\title{
Quantum Logic, State Space Geometry and Operator Algebras
}

\author{
L. J. Bunce and J. D. Maitland Wright \\ The University of Reading, Department of Mathematics, Whiteknights, Reading, RG6 2AX, United \\ Kingdom
}

\begin{abstract}
The problem of characterising those quantum logics which can be identified with the lattice of projections in a JBW-algebra or a von Neumann algebra is considered. For quantum logics which satisfy the countable chain condition and which have no Type $I_{2}$ part, a characterisation in terms of geometric properties of the quantum state space is given.
\end{abstract}

\section{Introduction}

Quantum logics, as defined below, are $\sigma$-complete orthomodular lattices. They have been vigorously investigated in recent years. In most mathematical formulations of the foundations of quantum mechanics the lattice of "questions" associated with a physical system is a quantum logic.

Important examples of quantum logics are, in order of successive generalisation:

(a) The lattice of all closed subspaces of a separable Hilbert space.

(b) The lattice of all projections in a von Neumann algebra.

(c) The lattice of all projections in certain Jordan operator algebras known as JBW-algebras.

Characterisation of those quantum logics isomorphic to (a) have been obtained by Piron, in 1964, (see [8]), and by Wilbur [9], in 1977. Can one characterise those quantum logics isomorphic to the lattice of all projections in a von Neumann algebra, or in a JBW-algebra, by geometric properties of the quantum state space of a quantum logic?

We obtain a partial solution to this problem by restricting our attention to quantum logics which satisfy the countable chain condition and which have no Type $I_{2}$ part (see below for definitions). We show that, when $Q$ is such a quantum logic, there are three geometric properties which will be satisfied by the quantum state space of $Q$ if, and only if, $Q$ is isomorphic to the lattice of all projections in a JBW-algebra.

We also, as a corollary, give a geometric characterisation of those orthomodular 
lattices which are isomorphic to the projection lattice of a countably decomposable von Neumann algebra with no Type $I_{2}$ direct summand.

Let $L$ be an orthomodular lattice with orthocomplementation $x \mapsto x^{\perp}$. A probability measure, $\phi$, on $L$ is a non-negative real valued function, $\phi: L \rightarrow \mathbb{R}_{+}$, such that $\phi(0)=0, \phi(1)=1$, and if $\left(x_{n}\right)$ is a sequence, in $L$, of mutually disjoint elements for which $\vee x_{n}$ exists, then $\phi\left(\vee x_{n}\right)=\Sigma \phi\left(x_{n}\right)$. (The sequence $\left(x_{n}\right)$ is said to be mutually disjoint if $x_{n} \leqq x_{m}^{\perp}$, for every $m, n$, with $m \neq n$.) The set of all probability measures on $L$ is a convex set which we shall denote by $K_{L}$.

The convex set $K_{L}$ is said to be strongly full if the following three properties are satisfied.

(1) For $x, y \in L$ we have $x \leqq y$ if

$$
\left\{\phi \in K_{L}: \phi(x)=1\right\} \subseteq\left\{\phi \in K_{L}: \phi(y)=1\right\} .
$$

(2) Whenever $x, y \in L$ and $\phi \in K_{L}$ with $\phi(x)=\phi(y)=1$, then $\phi(x \wedge y)=1$.

(3) Whenever $\phi$ lies in a proper norm-exposed face of $K_{L}$, then $\phi(x)=0$ for some non-zero element $x$ of $L$. (A face $F$ of $K_{L}$ is said to be norm-exposed if there exists a bounded affine function, $b$, on $K_{L}$ such that $b>0$ on $K_{L} \backslash F$ and $b=0$ on $F$.)

The orthomodular lattice, $L$, is said to satisfy the countable chain conditions (abbreviated c.c.c.) if every family of mutually disjoint elements in $L$ is at most countable. It is said that $L$ is a quantum logic if $\vee x_{n}$ exists in $L$ whenever the sequence $\left(x_{n}\right)$, of elements of $L$, is mutually disjoint. It is easy to see that a quantum logic which satisfies the c.c.c. is a complete orthomodular lattice.

Consider the orthomodular lattice $L$ and let $x$ be an element of $L$. The order interval, $L[0, x]=\{y \in L ; y \leqq x\}$, is an orthomodular sublattice of $L$ with the complementation $y \rightarrow x \wedge y^{\perp}$. The element $x$ of $L$ is said to be abelian if $L[0, x]$ is distributive. The elements $y$ and $z$ of $L$ are said to commute if $y$ and $z$ generate a distributive sublattice of $L$. The set of all those elements of $L$ which commute with every other element of $L$ is said to be the centre, $Z(L)$, of $L$. It is said that $L$ is factor if $Z(L)=\{0,1\}$.

If $L$ is a complete orthomodular lattice, then so is $Z(L)([5],[8])$ and, consequently, for each $x$ in $L$ we can define the central support of $x$ in $L$ :

$$
c(x)=\wedge\{y \in Z(L) ; x \leqq y\} \in Z(L) .
$$

We say that the complete orthomodular lattice, $L$, has Type $I_{2}$ part if there exist, in $L$, disjoint non-zero abelian elements $x, y$ such that $x \vee y=c(x)=c(y)$; is disjoint abelian elements $x, y$ can be chosen so that $x \vee y=c(x)=c(y)=1$, then $L$ is said to be of Type $I_{2}$.

Recall that the convex set, $F$, is said to be spectral if it is the base of a base-norm space, $(V, F)$, and $(V, F)$ is in spectral duality (see $\left[1\right.$, Sects. 6, 7]) with $A^{b}(F) \simeq V^{*}$, where $A^{b}(F)$ represents the bounded affine functions on $F$. The spectral convex set $F$ is elliptic if $P\left(Q-Q^{\prime}\right) P^{\prime}=0$ for all $P$-projections $P, Q$ of $A^{b}(F)\left(Q^{\prime}\right.$ represents the quasi-complement of the $P$-projection $Q,[1])$.

Iochum and Schultz [6] have shown that a convex set is (affinely isomorphic to) the normal state space of a JBW-algebra if and only if it is spectral and elliptic. 
Theorem. Let $L$ be a quantum logic satisfying the countable chain condition with no Type $I_{2}$ part. Then Lis isomorphic to the lattice of all projections in a JBW-algebra if and only if $K_{L}$ is strongly full, spectral and elliptic.

Proof. Suppose that $K=K_{L}$ is strongly full, spectral and elliptic. Given an element $x$ in $L$, define the element $\hat{x}$ of $A^{b}(K)^{+}$, by $\hat{x}(\lambda \phi+(1-\lambda) \psi)=\lambda \phi(x)+(1-\lambda) \psi(x)$, $\lambda \in[0,1], \phi, \psi \in K$. Notice then that condition (1) in the definition of strongly full implies that the map $L \rightarrow \hat{L}=\{\hat{x}: x \in L\}$ is an order isomorphism and that $\hat{L}$ is an orthomodular lattice, isomorphic to $L$, with the lattice operations defined by $\hat{x} \vee \hat{y}=$ $(x \vee y)^{\wedge}, \hat{x} \wedge \hat{y}=(x \wedge y)^{\hat{\gamma}},(\hat{x})^{\perp}=\left(x^{\perp}\right)^{\wedge}$. We may therefore suppose that $L$ is contained in $A^{b}(K)^{+}$and that $x=\hat{x}$, for each $x$ in $L$. Observe that (with the above identification) 1 is the order unit of $A^{b}(K)$, and that for $x, y$ in $L$,

$$
x^{\perp}=1-x ; x \vee y=x+y, \quad \text { if } x \leqq y^{\perp} ; x \wedge y^{\perp}=x-y, \quad \text { if } y \leqq x .
$$

Furthermore, since $K$ is spectral and elliptic, we can identify $A^{b}(K)$ with a JBWalgebra, $M$, which has normal state space $K$, by the result of Iochum and Schultz, [6, Theorem 1.5], mentioned above.

Let $\phi \in K$. The condition (2), in the definition of strongly full, implies that the set $\{x \in L ; \phi(x)=1\}$ is downward directed. Since $L$ is a complete lattice, this means that $\phi\left(s_{L}(\phi)\right)=1$, where $s_{L}(\phi)=\wedge\{x \in L ; \phi(x)=1\}$, the support of $\phi$ in $L$. Let $\left(\phi_{i}\right)_{i \in I}$ be a maximal family in $K$ for which the $s_{L}\left(\phi_{i}\right)$ are mutually disjoint. If $I$ is infinite, then we can take $I=\mathbb{N}$, since $L$ satisfies the c.c.c.. It is easy to check that condition (1) implies that for each non-zero $y$ in $L$ there exists $\psi$ in $K$ such that $\psi(y)=1$. It follows from this that $\Sigma s_{L}\left(\phi_{n}\right)=1$. Now with $\phi=\Sigma\left(1 / 2^{n}\right) \phi_{n}$, we see that $s_{L}(\phi)=1$ (when $I$ is finite, the proof of the existence of such $\phi$ is similar). Observe now that condition (3) implies that $\phi$ is a faithful normal state on $M$. In addition, since a JBW-algebra has a faithful normal state if and only if its lattice of projections satisfies the c.c.c. (the proof is similar to the usual $W^{*}$-proof, see [7, II.3.19]), it follows that every projection in $M$ is the support projection of some normal state on $M$.

The range projection, $r(a)$, of an element $a$ in $M^{+}$is the projection in $M$ which is the unit element of the hereditary JBW-subalgebra of $M$ generated by $a$. We note that for each $x$ in $L, 1-r(1-x) \leqq x \leqq r(x)$. In addition, since $\phi(a)=0$ if and only if $\phi(r(a))=0, a \in M^{+}, \phi \subset K$, it follows from (1) that the following three conditions are equivalent for elements $x, y$ of $L$ : (i) $x \leqq y$; (ii) $r(x) \leqq r(y)$; (iii) $x \leqq r(y)$. In particular, for $x$ in $L, r(x)=1$ if and only if $x=1$.

Let now $p$ belong to $\mathbb{P}$, the projection lattice of $M$, such that $p \neq 0,1$. By the above remarks, there exist $\phi$ in $K$ such that $p=s(\phi)$ - the support projection of $\phi$ in $\mathbb{P}$. Since $\phi$ then lies in a proper norm exposed face of $K$, there exists $x$ in $L, x \neq 1$, with $\phi(x)=1$. But then, $\phi(1-r(1-x))=1$, and so $p=s(\phi) \leqq 1-r(1-x) \leqq x$.

It follows that $L \subseteq \mathbb{P}$. Indeed, let $y \in L$. Then $r(y) r(1-y) \neq 1$. Suppose that $q=r(y) r(1-y) \neq 0$. Then, it follows from the preceding paragraph that there exist a non-zero $x$ in $L$ such that $x \leqq q \leqq r(y), r(1-y)$. Consequently, $x \leqq y$ and $x \leqq 1-y$, implying that $x=0$, a contradiction. Hence, $r(y) r(1-y)=0$, which implies that $y(1-y)=0$. Therefore $y$ is a projection.

Let $\underline{v}$ denote lattice suprema in $\mathbb{P}$. Then, for $x, y$ in $L, x \underline{\vee} y \leqq x \vee y$. On the other hand, given $\phi$ in $K$, if $\phi(x \underline{\vee} y)=0$, then $\phi(x)=\phi(y)=0$, so $\phi(x \vee y)=0$, by (2). Hence, by (1), $x \vee y \leqq x \underline{\vee}$. It follows that $L$ is a sublattice of $\mathbb{P}$. 
Finally, since, as we have seen, given $p \in \mathbb{P}, p \neq 0$, there exist non-zero elements of $L$ dominated by $p$, we can choose a maximal mutually disjoint family $\left\{x_{\alpha}\right\}$ in $L$ dominated by $p$. Then, for each $\phi$ in $K_{L}, \phi\left(\vee x_{\alpha}\right)=\Sigma \phi\left\{x_{\alpha}\right\}=\phi\left(\Sigma x_{\alpha}\right)$. So, $\vee x_{\alpha}=$ $\Sigma x_{\alpha} \leqq p$ and hence $\vee x_{\alpha}=p$, by maximality. Consequently, $L=\mathbb{P}$.

To obtain the converse we shall make essential use of the results of [3] which generalizes the work of Christensen [4] and Yeadon [10, 11].

Let us now suppose that $L$ is isomorphic to the projection lattice $\mathbb{P}$, of a JBWalgebra, $M$. Then $K=K_{L}$ is affinely isomorphic to the set of probability measures (as defined here) on $\mathbb{P}$, also denoted by $K$. From [3, Lemma 3.5(iii)], for example, we see that $M$ has no Type $I_{2}$ direct summand. Therefore, since $\mathbb{P}$ satisfies the c.c.c., it follows from [3, Corollary 5.5] that $K$ can be identified with the normal state space of $M$. Therefore, as can be seen from the results of [1], $K$ is strongly full for $\mathbb{P}$ and, by [6, Theorem 1.5], we know that $K$ is spectral and elliptic. This completes the proof.

By [6, Theorem 2.9], the normal state space of a JBW-algebra $M$ has the global 3-ball property if, and only if, $M$ is the self-ajoint part of a von Neumann algebra.

This observation and the above theorem gives the following corollary.

Corollary. A quantum logic L which satisfies the countable chain condition and with no Type $I_{2}$ part is isomorphic to the projection lattice of a von Neumann algebra if and only if $K_{L}$ is strongly full, spectral, elliptic and has the global 3-ball property.

\section{References}

1. Alfsen, E. M., Shultz, F. W.: Non-commutative spectral theory for affine function spaces on convex sets. Mem. Am. Math. Soc. 172 (1976)

2. Alfsen, E. M., Shultz, F. W.: On non-commutative spectral theory and Jordan algebras. Proc. London Math. Soc. 38, 497-516 (1979)

3. Bunce, L. J., Wright, J. D. M.: Quantum measures and states on Jordan algebras. Commun. Math. Phys. (to appear)

4. Christensen, E.: Measures on projections and physical states. Commun. Math. Phys. 86, 529-538, (1982)

5. Holland, S. S. Jr.: A Radon-Nikodym theorem in dimension lattices. Trans. Am. Math. Soc. 108, 67-87, (1963)

6. Iochum, B., Schultz, F. W.: Normal state spaces of Jordan and von Neumann algebras. J. Funct. Anal. 50, 317-328 (1983)

7. Takesaki, M.: Theory of operators I. Berlin. Heidelberg, New York: Springer 1979

8. Varadarajan, V. S.: Geometry of quantum theory. Vol. I. Amsterdam: van Nostrand 1968

9. Wilbur, W. J.: On characterising the standard quantum logics. Trans. Am. Math. Soc. 233, 265-282 (1977)

10. Yeadon, F. J.: Measures on projections in $W^{*}$-algebras of Type II. Bull. London Math. Soc. 15, 139$145(1983)$

11. Yeadon, F. J.: Finitely additive measures on projections in finite $W^{*}$-algebras. Preprint

Communicated by H. Araki

Received February 1, 1984 